\title{
6. SIMULTANEOUS X-RAY, OPTICAL AND RADIO OBSERVATIONS OF GALACTIC X-RAY SOURCES
}

\author{
W. A. HILTNER \\ University of Michigan, Ann Arbor, Mich., U.S.A.
}

\begin{abstract}
Simultaneous observations of the X-ray source Sco X-1 are reviewed and discussed. Several conclusions can be drawn from the observations. (a) There is no correlation of the radio intensities with either the optical or X-ray intensities. (b) The X-ray intensity is high and variable only when the blue optical magnitude is brighter than 12.6-12.7. (c) There is often correlation of $\mathrm{X}$-ray and optical flares, but the relationship is not one to one.

Attention is called to the paucity of simultaneous observations of other identified X-ray sources.
\end{abstract}

In order to make simultaneous $\mathrm{X}$-ray, optical and radio observations it is obvious that the X-ray source must be identified in other spectral regions of interest. This requirement has emerged as one of the principal obstacles for more extensive observations in spectral regions other than the X-ray. However, as will become apparent, it is not the only one.

The number of galactic X-ray sources, excluding the old novae, that have reliable identifications in other spectral regions are very few. In the optical region Sco X-1, Tau X-1 (the Crab Nebula) and Cyg X-2 are identified with confidence. Cyg X-1 and 2U 0900-40 may be associated with optical spectroscopic binaries (Bolton, 1972; Webster and Murdin, 1972; and Hiltner and Osmer, 1972), the secondary components of which may possibly be the X-ray object. Both sources are variable in X-ray intensity (Giaconni et al., 1972) and both spectroscopic binaries have been reported as probable variables in the optical region (Kukarkin and Soluyanov, 1972; and Hiltner et al., 1972). The two X-ray sources for which observations at other wavelengths should prove most exciting are Cen X-3 and Her X-1. Both are eclipsing binaries (Schreier et al., 1972) as observed in the X-ray region. Neither has been identified in the optical or radio. Their identification should receive the highest priority.

The need for simultaneous observations at different spectral regions implies that the sources are variable in more than one region and non-periodic. It now seems relatively safe to assume that most, if not all, $\mathrm{X}$-ray sources are variable. The three galactic sources listed above with reliable optical and radio identifications all are variable in intensity at both X-ray and optical wavelengths and two are known to be variable at radio frequencies. Simultaneous observations can therefore possibly assist in developing a model for the X-ray stars.

Only Sco X-1 has been observed simultaneously at two or more spectral regions, and these observations will be discussed in some detail, after a few comments on Cyg X-2. Kristian et al. (1967) showed that Cyg X-2 was variable in optical intensity and spectroscopic studies (Kraft and Demoulin, 1967; and Kraft and Miller, 1969) of the optical counterpart have been published. However, there have been no simul- 
taneous observations reported in the literature. The neglect of this object by optical astronomers is no doubt a consequence of its faintness. Its B magnitude is 15.2, a factor of 5 to 10 fainter than the optical counterpart of Sco X-1. No attempt will be made to discuss Tau X-1, the Crab Nebula, but I refer the reader to the many reviews and extensive literature on this object. Furthermore, the variable optical component is periodic.

Our discussion by necessity will therefore be centered around Sco X-1 of which there are now sufficient simultaneous observations for analysis. Before discussing the simultaneous observations it is useful to review the independent observations because of their much greater abundance.

At the time of its optical identification in 1966 Sandage et al. (1966) showed that the optical counterpart was highly variable in light intensity. Subsequent extensive observations (Hiltner and Mook, 1967, 1970; Westphal et al., 1968) showed irregular light variations with the following characteristics: (1) There are often night to night

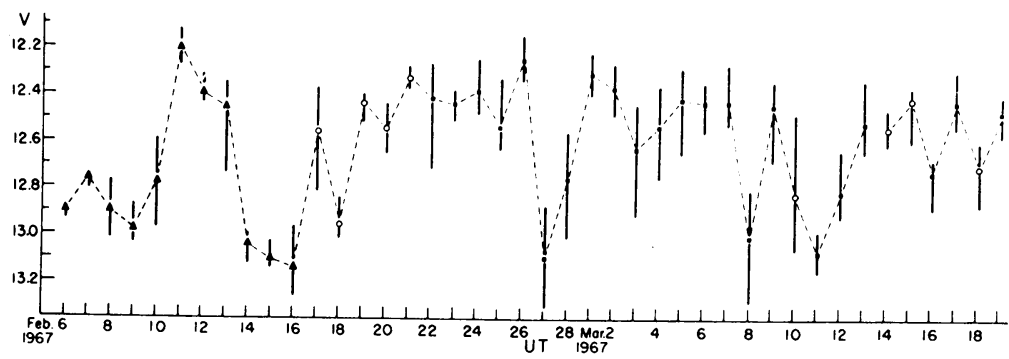

Fig. 1. Average visual magnitude of Sco X-1 for 42 consecutive nights. (Mook, 1967). Bars through the data points represent the total range in $V$ over which Sco X-1 varied on that night.

variations of a magnitude, (2) smooth variations of one-half magnitude in an hour or two, (3) flares with an amplitude of about $0.2 \mathrm{mag}$ and a rise time as short as $90 \mathrm{~s}$, and (4) a flickering with an amplitude of about $0.02 \mathrm{mag}$ on a time scale of a minute, more or less. The first three types of variations are shown in Figures 1 and 2 and the last in Figure 3. It has been shown by Westphal et al. (1968) that the flickering is superimposed on all the other types of variations listed above. No periodicity has been established although transient oscillations have been reported after the object has flared (see Figure 3).

Rocket flights have shown that the X-ray intensity of Sco X-1 in the 2 to $10 \mathrm{keV}$ range is variable, perhaps by a factor of two. The first $\mathrm{X}$-ray flare was observed in 1967 by Lewin et al. (1968). During this balloon flight the intensity of hard X-rays was observed to increase (Figure 4) by a factor of four in ten minutes. The high X-ray intensity then decayed with a characteristic time of about thirty minutes. Further balloon flights by Lewin et al. (1970) confirm these earlier observations of large variations in X-ray intensity. Changes in X-ray intensity on a shorter time scale have been observed by Evans et al. (1970) as shown in Figure 5. 

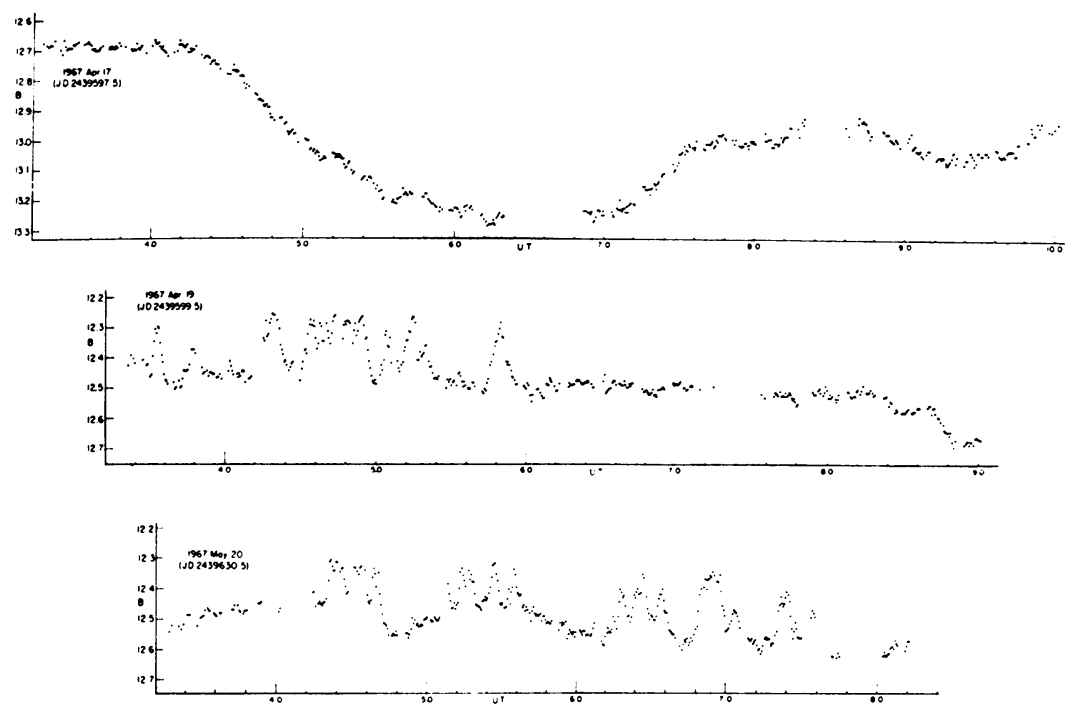

Fig. 2. Representative light curves for Sco X-1 (Hiltner and Mook, 1967).

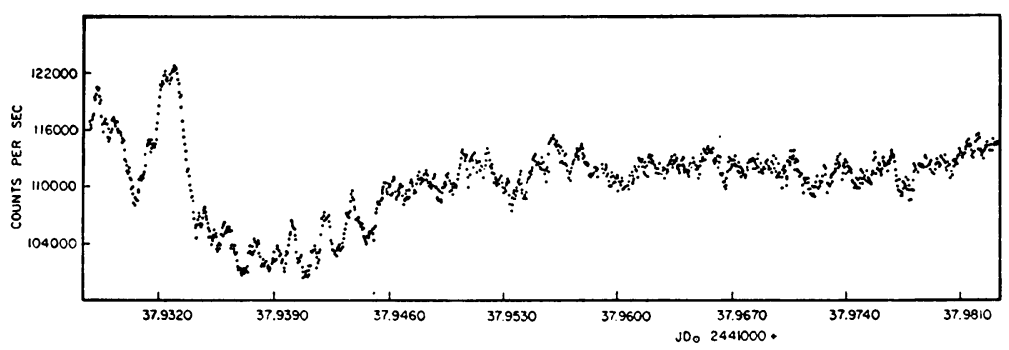

Fig. 3. Portion of the light curve of Sco X-1 on 1971 March 27 (Robinson and Warner, 1972). Each point represents an integration for $4 \mathrm{~s}$. Note the 'oscillations' that persisted for about $15 \mathrm{~min}$ and then faded.

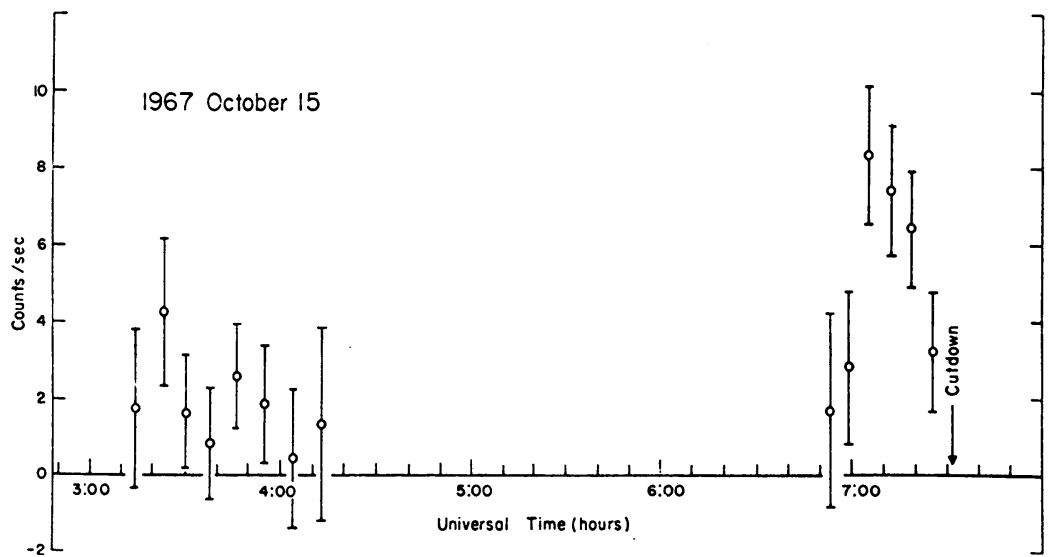

Fig. 4. Intensity of $\mathrm{X}$-rays from Sco $\mathrm{X}-1$ in the $20-30 \mathrm{keV}$ range as a function of universal time on 1967 October 15 (Lewin et al., 1968). 


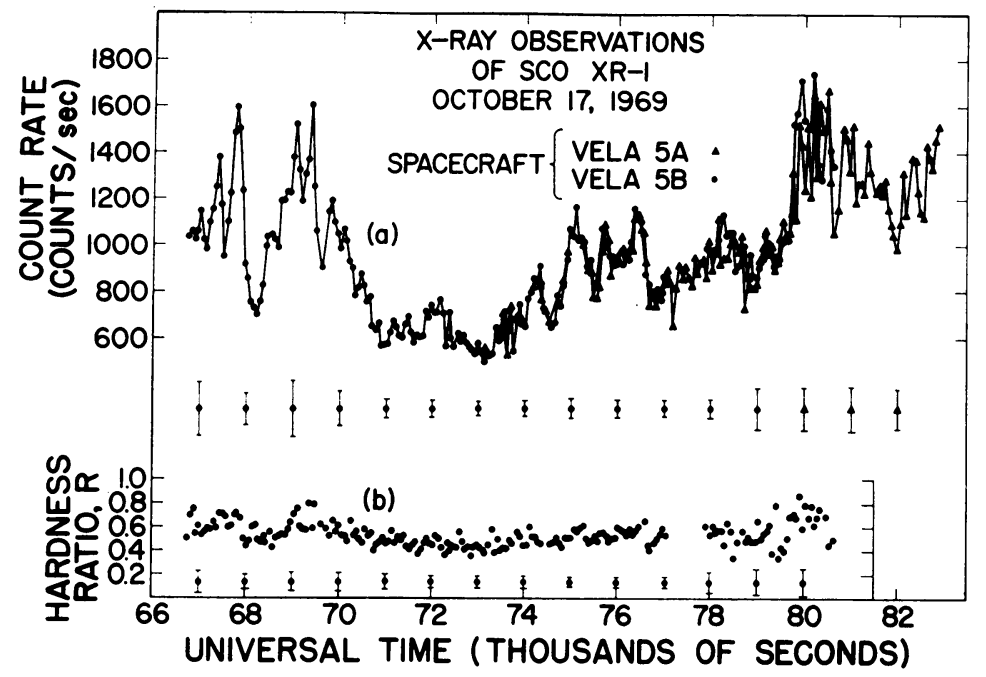

Fig. 5. X-ray observations of Sco X-1 on 1969 October 17. (a) Observed 3-12 keV counting rate versus time and (b) spectral hardness ratio versus time. Typical error bars are shown below each curve (Evans et al., 1970).

Radio observations by Hjellming and Wade (1971) have shown that Sco X-1 consists of a highly variable source centered on the optical counterpart and two satellite sources on opposite sides of the central source and probably constant in intensity. This configuration has been confirmed by Braes and Wiley (1972). Further observations by Wade and Hjellming (1972) showed from about $300 \mathrm{~h}$ of observing that major flaring of the central source was in progress $15 \%$ of the time, dormant $(<0.003$ flux units, the limit of detection) $20 \%$, constant at about 0.01 flux units $5 \%$ and for the remaining $60 \%$ the object was relatively weak ( 0.01 to 0.03 flux units) but erratically and continuously variable.

The need for simultaneous X-ray, optical and radio observations is obvious for Sco $\mathrm{X}-1$ is highly variable in all wavelengths and non-periodic.

The earliest attempt to make simultaneous observations has been reported by Burginyon et al. (1970). Eight rocket flights were made while the optical counterpart of Sco X-1 was monitored for blue magnitude. The eight X-ray observations were made in the B magnitude interval from 13.2 to 12.4. Some correlation of the X-ray intensity and the B magnitude can be seen in Figure 6, but the uncertainties in the observations are only a little smaller than the effect.

The OSO-3 and Vela satellites gave the earliest opportunity to obtain more detailed correlations of X-ray and optical intensities of Sco X-1. Hudson et al. (1970) observed two X-ray flares in Sco X-1 for which there was also optical coverage. These flares, one of which is shown in Figure 7 (June 3), correlated nicely at the two spectral region. The X-ray enhancement amounted to about a factor of two over the quiescent emission in the 7.7 to $12.5 \mathrm{keV}$ energy range.

These correlations have been confirmed by further observations by Pelling (1972) 


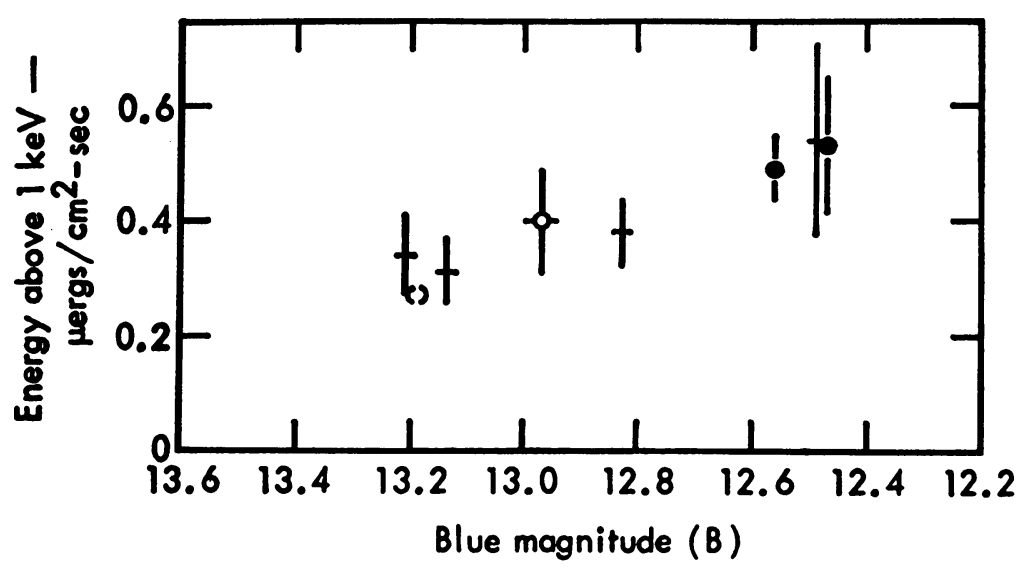

Fig. 6. Results of simultaneous X-ray and optical observations of Sco X-1 for eight rocket flights (Burginyon et al., 1970).

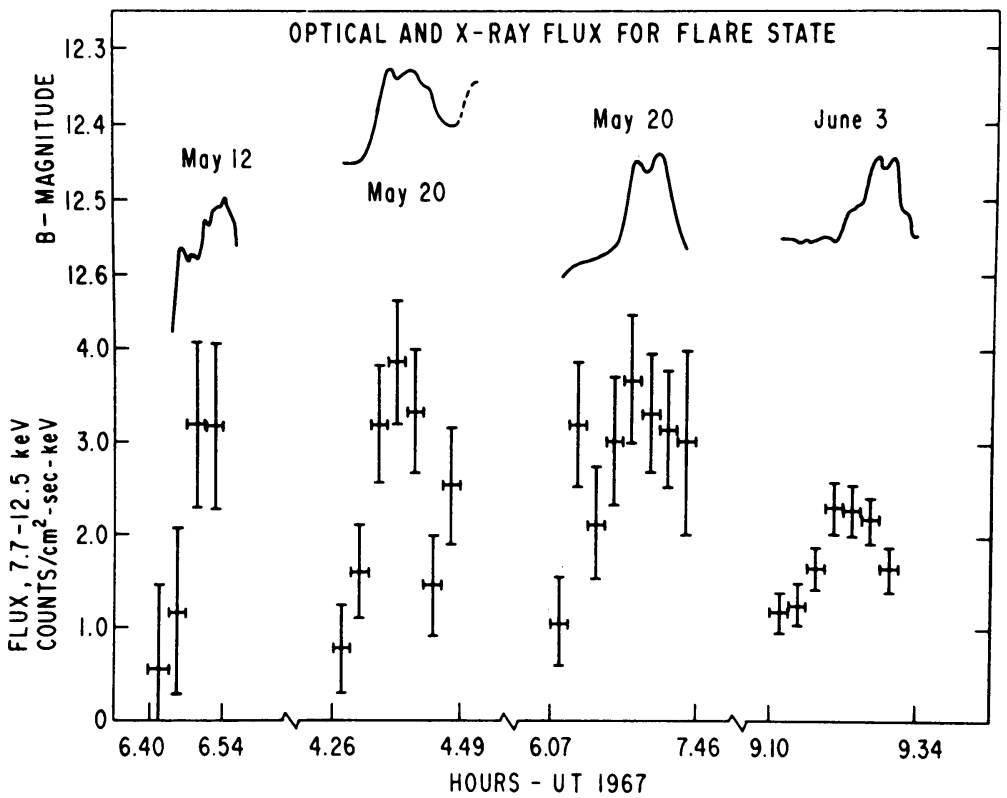

Fig. 7. Simultaneous optical and X-ray data for four flare episodes (Pelling, 1972). The night of June 3 was reported by Hudson et al. (1970).

who reported three additional X-ray flares observed by OSO-3 that were correlated with optical flares. They are also shown in Figure 7. A correlation of optical and X-ray flares was also confirmed by Evans et al. (1970). Their observations are shown in Figure 8. The X-ray intensity increase is significantly greater than that of the optical, a factor of two versus about $20 \%$.

The correlation outside of flaring may be very different. The OSO-3 observations 
(Pelling, 1972) are shown in Figure 9. The two cases shown in this figure differ only in assumed aspect of the satellite, the extremes, so that the true X-ray flux probably falls somewhere between. In Figure 10 are shown the Vela satellite observations obtained during the World Wide Watch of Sco X-1 in 1970. (Details will be published elsewhere.)

In both figures the data suggest a general decrease in X-ray flux as the object brightens optically until the magical magnitude $12.6-12.7$ is reached - the magnitude from which the object may (but not necessarily) flare in the optical range. This weak anti-correlation can also be seen in Figure 8. Further observations are needed to confirm or reject the reality of this slight anti-correlation.

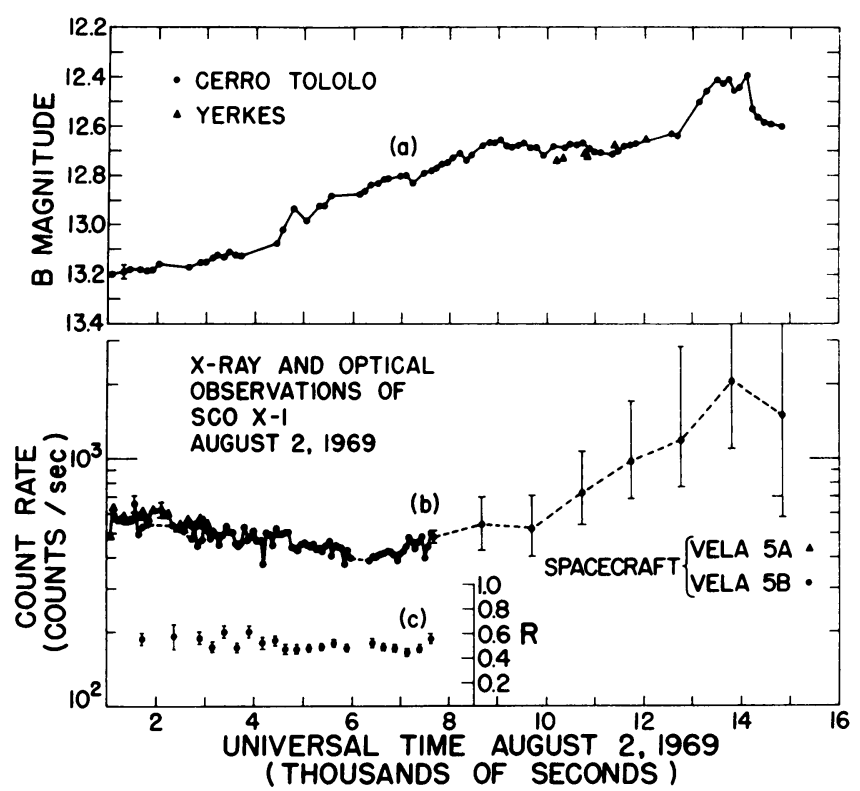

Fig. 8. Simultaneous X-ray and optical observations of Sco X-1. (a) B magnitude versus time, (b) 3-12 keV X-ray counting rate observed by the Vela 5 satellites and (c) ratio of counting rate in the two energy channels 6-12 and 3-6 keV. After $8000 \mathrm{~s}$ the error bars represent upper and lower limits of the counting rate (Evans et al., 1970).

Kastenbaum et al. (1971) have looked for correlation of X-ray and optical flickering. Their results from a rocket flight are shown in part in Figure 11. The two power spectra for the two spectral regions show a strong maximum at a period of $20 \mathrm{~s}$. The correlation of the oscillations persisted for about two minutes.

The X-ray satellite SAS-A (UHURU), was programmed so that during the period March 24 to 28, 1971, Sco X-1 was observed by the satellite while optical and radio observations were made from ground-based observatories. The optical and radio coverage was for a longer period. The optical observations were made at Cerro Tololo Inter-American Observatory, Leiden Southern Station, the University of Michigan and the University of Texas and the radio observations were made at National Radio 
Astronomy Observatory and Westerbork*. The observations from March 22 to April 2, 1971 are shown in Figure 12. During these twelve days there was flare activity in both the optical and radio observations as well as periods of quiescence. The optical

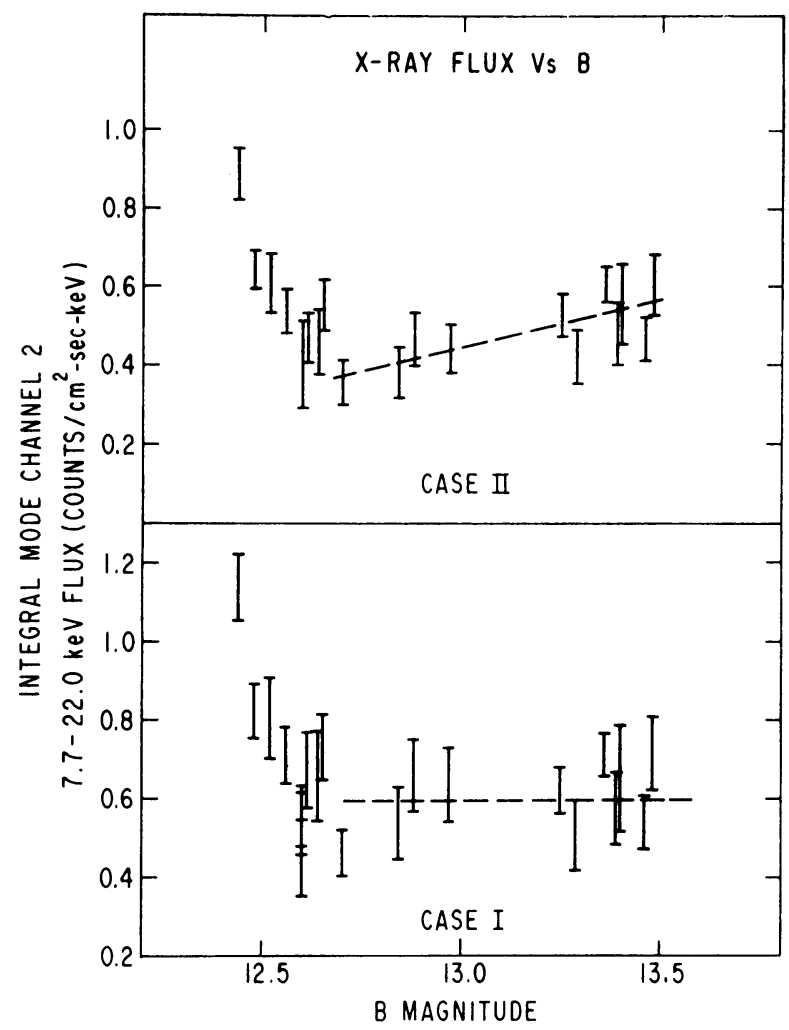

Fig. 9. X-ray intensity versus B magnitude (Pelling, 1972). The two curves differ in assumed satellite aspect; the opposite extremes.

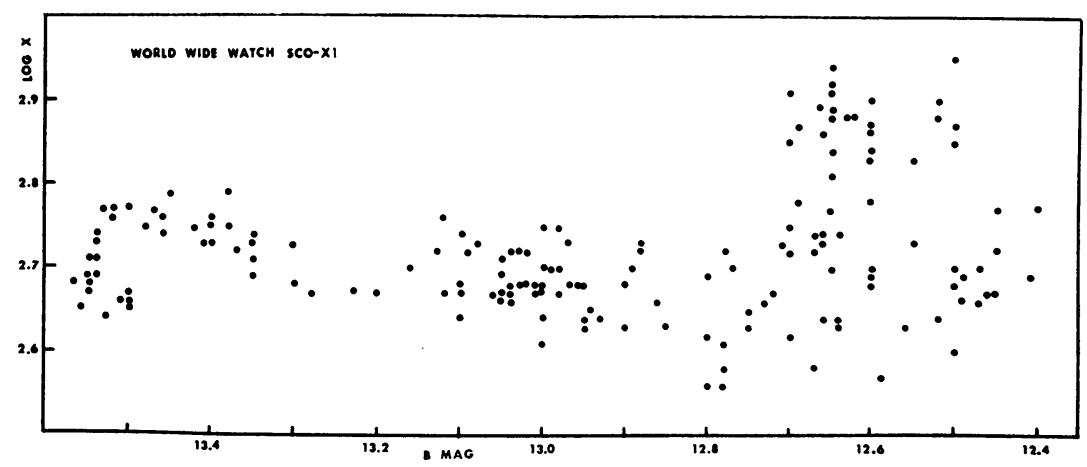

Fig. 10. X-ray intensity versus B magnitude from the 'World Wide Watch' of Sco X-1 in 1970. Details to be published elsewhere.

* Participating observers included H. Bradt, L. Braes, W. Hiltner, R. Hjellming, W. Kunkel, G. Miley, J. Pel, J. Thomas, P. Vanden Bout, C. Wade and B. Warner. 
intensity was always low when there was radio flaring and on occasion both spectral regions were faint simultaneously. During optical flaring the radio emission was in the 'dormant' or 'relatively weak' state. Judging from these observations apparently there is no obvious correlation between the optical and radio intensities.

Figure 13 shows all the available observations, X-ray, optical and radio, for the period March 24-28, 1971. An inspection of the figure suggests the following. (1) When the object is faint optically it is also faint in the X-ray region. (2) When it is bright at optical wavelengths it is bright and active in X-ray. (3) No correlation between $\mathrm{X}$-ray and radio is apparent.

A close inspection of the figure on the night of March 26 will reveal a rapid increase in optical brightness followed an hour later by a rapid increase in X-ray intensity. One must not conclude that this represents a related phenomenon but rather only the

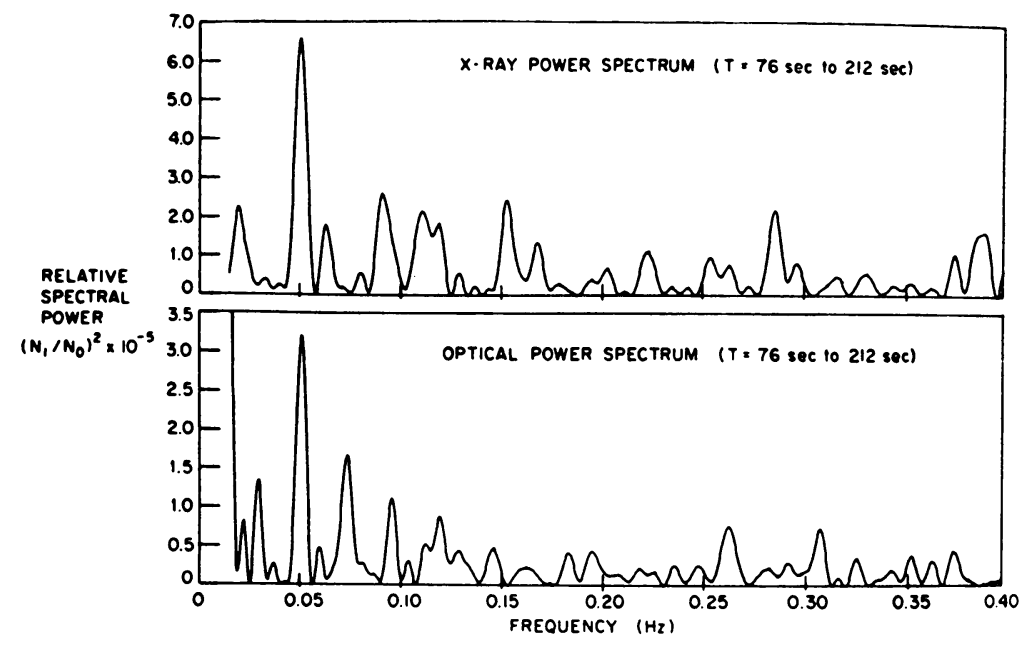

Fig. 11. The power spectrum of Sco X-1 in the X-ray and optical spectral regions for a duration of $136 \mathrm{~s}$ (Kestenbaum et al., 1971). Note the large increase in power at both wavelengths at $0.05 \mathrm{~Hz}$ (20 s period).

absence of optical coverage during the rise in X-ray intensity. Note that Sco X-1 had just reached the critical magnitude from which it may flare when the optical coverage was discontinued and the X-ray intensity was still low.

Figures 14 and 15 show the nights of October 27 and 28 respectively in more detail. These figures clearly show that the optical and X-ray flares are related, but not one to one. The decrease in X-ray intensity near 10.5 UT on March 27 with no change in optical intensity is very striking while other changes are nicely correlated. Note that on these two nights Sco $\mathrm{X}-1$ was relatively faint and quiet in the radio region.

Figure 16 shows the late October to early November observations of Sco X-1 made by OSO-7 along with radio observations made at the National Radio Astronomy Observatory. These observations give beautiful confirmation that the radio and $\mathrm{X}$-ray intensities are unrelated. 


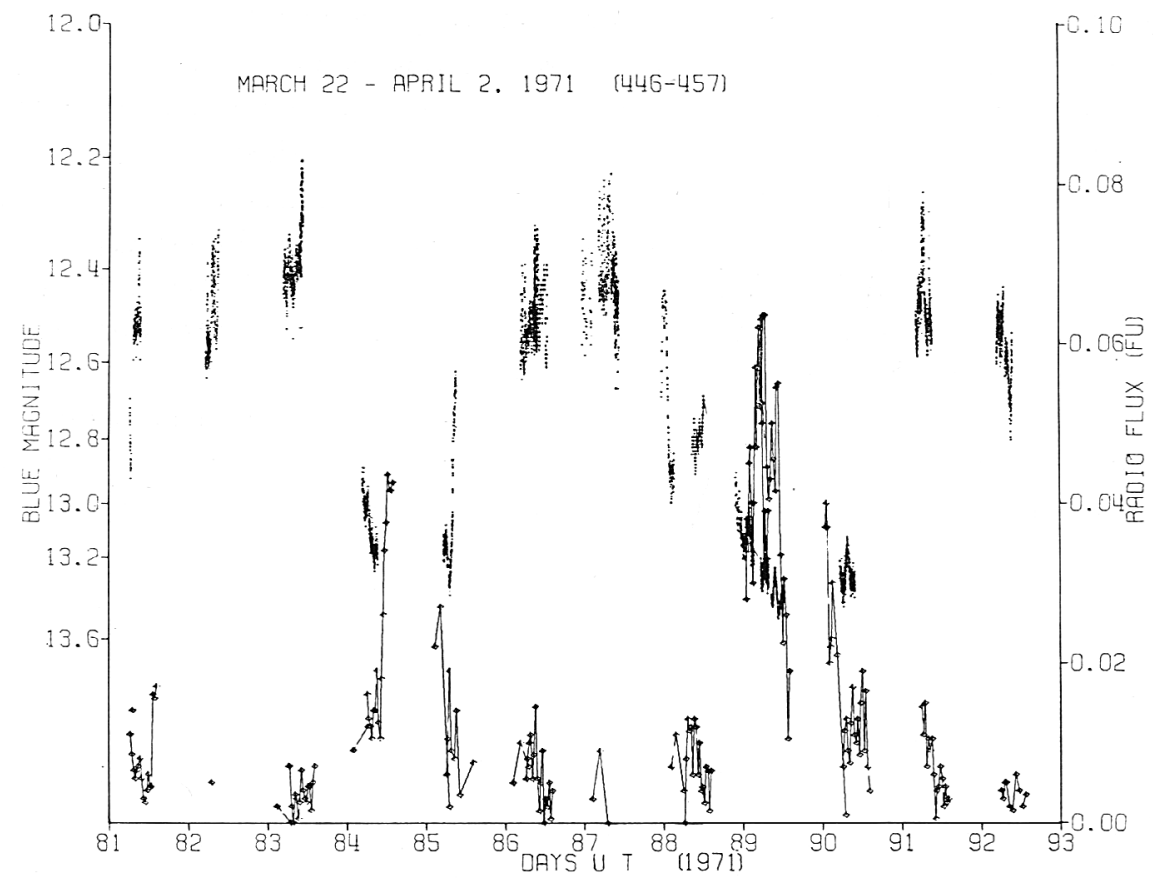

Fig. 12. Simultaneous optical and radio observations of Sco X-1 for the period 1971 March 22 to April 2.

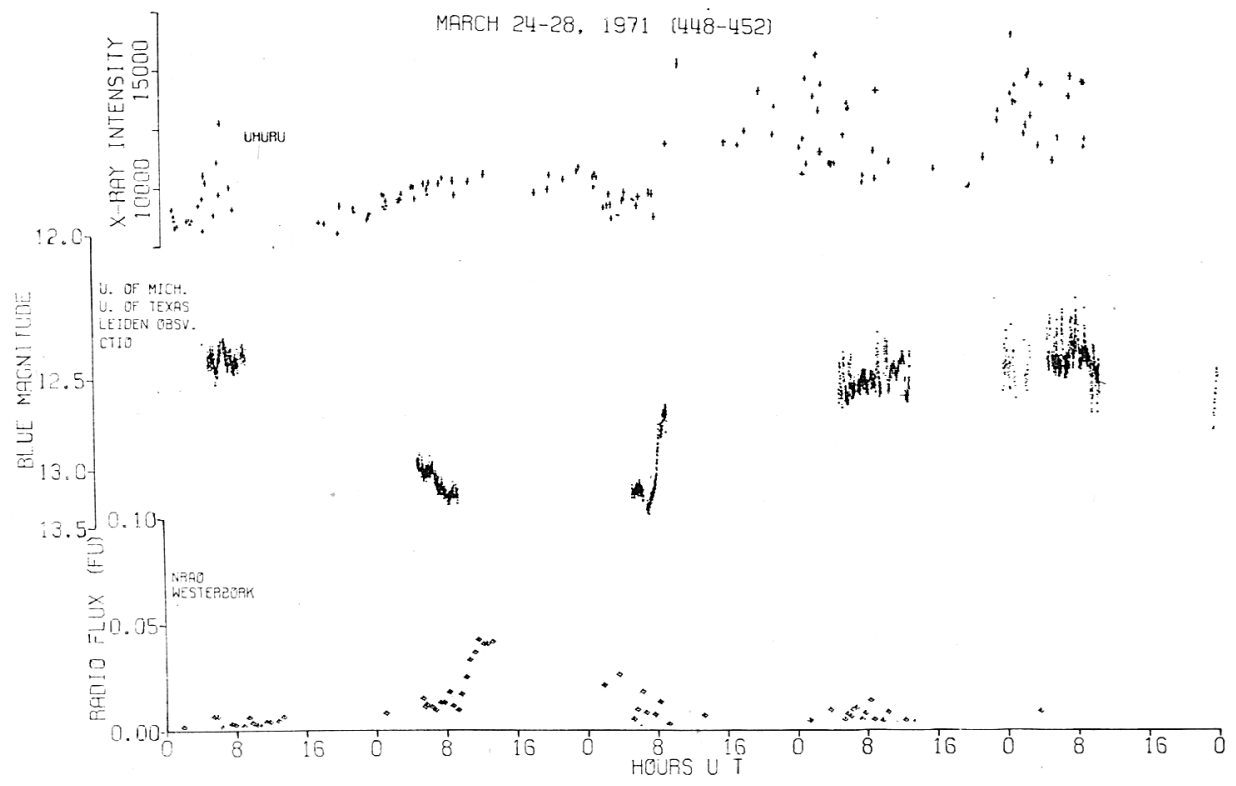

Fig. 13. Simultaneous X-ray, optical and radio observations of Sco X-1 from 1971 March 24 to March 28. 
In summary, the data now available strongly support the following conclusions: (a) There is no correlation of the radio intensities with either the optical or X-ray intensities. Thus far, the data show that whenever Sco X-1 has flared in the radio region it has been faint in the $\mathrm{X}$-ray and optical spectral regions and vice versa. (b) The X-ray intensity is high only when the blue optical magnitude is brighter

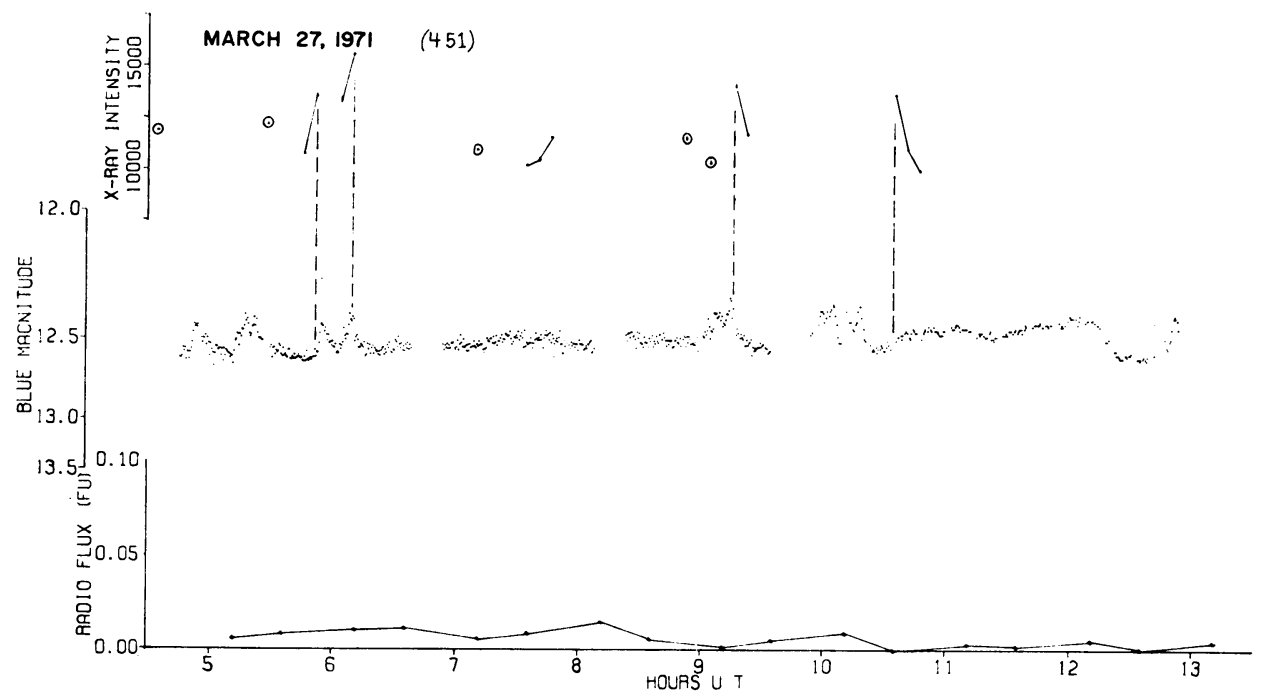

Fig. 14. Simultaneous X-ray, optical and radio observations of Sco X-1 for 1971 March 27. The vertical lines were drawn to aid in visual analysis.

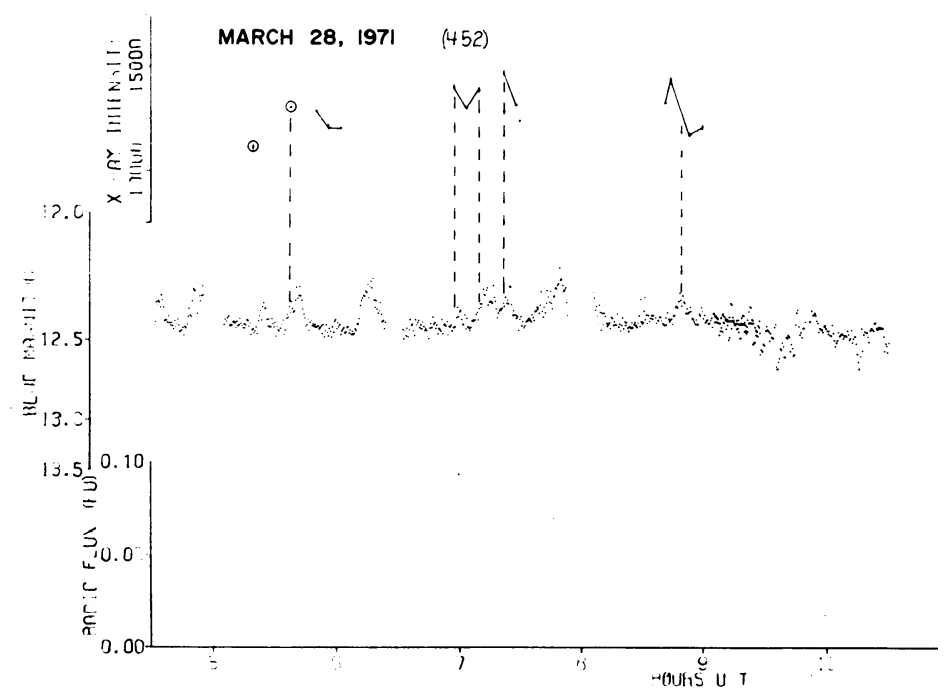

Fig. 15. Simultaneous X-ray, optical and radio observations of Sco X-1 for the night of 1971 March 28. The vertical lines are for visual aids. Preliminary estimates of the UHURU aspect errors yield an uncertainty in X-ray intensity of about $5 \%$. This is comparable to the smallest of the changes in intensity seen in Figures 14 and 15. 

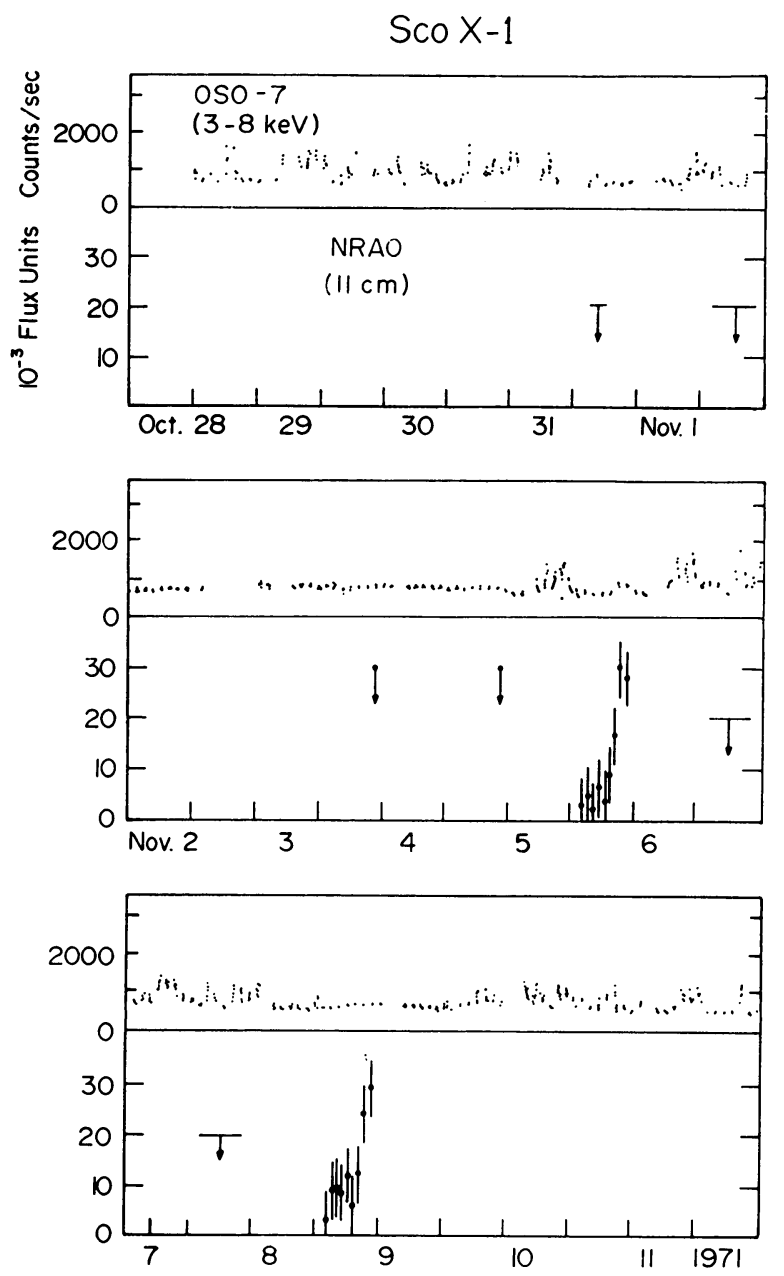

Fig. 16. Simultaneous X-ray and radio observations of Sco X-1 from 1971 October 28 to November 11.

than 12.6-12.7, the faintest magnitude from which the object will flare in the optical region.

(c) There may be a weak anti-correlation between the optical magnitude when Sco X-1 is fainter than 12.7 in B and the X-ray intensity, which is then always low (Figures 9 and 10).

(d) There is often correlation of X-ray and optical flares, but the relationship is not one to one. Significant changes in X-ray intensity have been observed to occur without an accompanying optical flare (e.g. Figure 14).

Further observations are always needed to more firmly establish the correlation of the X-ray and optical intensities. The absence of simultaneous radio and/or radio and optical flares needs further confirmation. Simultaneous X-ray and optical observations 
with good time resolution are needed in order to more firmly establish the correlation of flares in these two spectral regions. Then, simultaneous observation must be extended to other X-ray sources that can only lead to a better theoretical appreciation of these objects.

\section{References}

Bolton, C.: 1972, Nature 235, 271.

Braes, L. L. E. and Miley, G. K.: 1972, Astron. Astrophys., in press.

Burginyon, G. A., Grader, R. J., Hill, R. W., Price, R. E., Rodrigues, R., Seward, F. D., Swift, C. D., Hiltner, W. A., and Mannery, E. J.: 1970, Astrophys. J. $161,987$.

Evans, W. D., Belian, R. D., Conner, J. P., Strong, I. B., Hiltner, W. A., and Kunkel, W. E.: 1970, Astrophys. J. (Letters) 162, L115.

Giacconi, R., Murray, S., Gursky, H., Kellogg, E., Schreier, E., and Tananbaum, H.: 1972, Astrophys. J., in press.

Hiltner, W. A. and Mook, D. E.: 1967, Astrophys. J. 150, 851.

Hiltner, W. A. and Mook, D. E.: 1970, Astron. Astrophys. 8, 1.

Hiltner, W. A. and Osmer, P.: 1972, IAU Circ. No. 2398.

Hiltner, W. A., Werner, J., and Osmer, P.: 1972, Astrophys. J. Letters, in press.

Hjellming, R. M. and Wade, C. M.: 1971, Astrophys. J. Letters 164, L1.

Hudson, H. S., Peterson, L. E., and Schwartz, D. A.: 1970, Astrophys. J. Letters 159, L51.

Kestenbaum, H., Angel, J. R. P., Novick, R., and Cocke, W. J.: 1971, Astrophys. J. Letters 169, L49.

Kraft, R. P. and Demoulin, M. H.: 1967, Astrophys. J. Letters 150, L183.

Kraft, R. P. and Miller, J.: 1969, Astrophys. J. Letters 155, L159.

Kristian, J., Sandage, A., and Westphal, J.: 1967, Astrophys. J. Letters 150, L99.

Kukarkin, B. V. and Soluyanov: 1972, IAU Circ. No. 2395.

Lewin, W. H. G., Clark, G. W., and Smith, W. B.: 1968, Astrophys. J. Letters 152, L55.

Lewin, W. H. G., McClintock, J. E., Ryckman, S. G., Glass, I. S., and Smith, W. B.: 1970, Astrophys. J. Letters 162, L109.

Mook, D. E.: 1967, Astrophys. J. Letters 150, L25.

Pelling, R. M.: 1972, thesis.

Robinson, E. L. and Warner, B.: 1972, preprint.

Sandage, A. R., Osmer, P., Giacconi, R., Gorenstein, P., Gursky, H., Waters, J., Bradt, H., Garmire, G., Sreekantan, B., Oda, M., Osawa, K., and Jugaku, J.: 1966, Astrophys. J. 146, 316.

Schreier, E., Levinson, R., Gursky, H., Kellogg, E., Tananbaum, H., and Giacconi, R.: 1972, Astrophys. J. Letters 172, L79.

Wade, C. M. and Hjellming, R. M.: 1972, Astrophys. J., in press.

Webster, B. L. and Murdin, P.: 1972, Nature 235, 37.

Westphal, J., Sandage, A., and Kristian, J.: 1968, Astrophys. J. 154, 139. 